\title{
Double trouble or singular success: What can we expect from anatomic correction of congenitally corrected transposition of the great arteries?
}

\author{
Rohit S. Loomba, MD, ${ }^{a}$ and Andrew Redington, $\mathrm{MD}^{\mathrm{b}}$
}

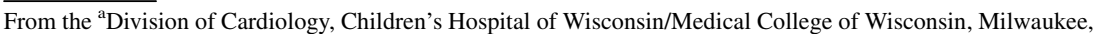
Wis; and the bivision of Cardiology, Cincinnati Children's Medical Center, Cincinnati, Ohio. Disclosures: Authors have nothing to disclose with regard to commercial support.

Received for publication March 22, 2017; accepted for publication March 23, 2017; available ahead of print April 14, 2017.

Address for reprints: Rohit S. Loomba, MD, 9000 Wisconsin Ave, Milwaukee, WI 53226 (E-mail: loomba.rohit@ gmail.com).

J Thorac Cardiovasc Surg 2017;154:266-7

$0022-5223 / \$ 36.00$

Copyright (c) 2017 by The American Association for Thoracic Surgery

http://dx.doi.org/10.1016/j.jtcvs.2017.03.104
}

The optimal repair strategy for patients with atrioventricular and ventriculoarterial discordance (congenitally corrected transposition of the great arteries [ccTGA]) remains a topic of debate. The article by Brizard and colleagues ${ }^{1}$ in this issue of the Journal is a timely reminder that the double switch and its variants provide a viable but far from perfect solution.

The Melbourne group ${ }^{1}$ shares its 32-patient, 19-year experience with anatomic correction for ccTGA. At 10 years, they describe a $19 \%$ combined incidence of death $(10 \%)$, transplant, or more than moderate left ventricular dysfunction and a 39\% incidence of reoperation; however, functional outcome was excellent in the survivors. Although these results are generally encouraging because of the complex nature of this population, they also highlight the significant morbidity and mortality relative to other congenital heart disease populations. These results also need to be placed in context with alternative strategies.

Anatomic repair offers the theoretic advantage of establishing the morphologically left ventricle as the systemic ventricle, with potential long-term advantages. Physiologic repair results in a systemic right ventricle, with its attendant risks of ventricular dysfunction and progressive tricuspid regurgitation (TR) in the long term. ${ }^{2}$ The choice superficially seems simple on that basis; however, the data from the Melbourne group ${ }^{1}$ remind us of the upfront "cost" of the anatomic repair relative to its sometimes more straightforward physiologic alternative. Indeed, the early and midterm mortalities for physiologic repair can be very low when there is minimal TR and no left ventricular outflow tract obstruction. ${ }^{2}$ Furthermore, the fact that not all patients with ccTGA, either without operation or after physiologic repair, will have right ventricular dysfunction and TR develop makes decision making for the individual patient even more difficult. Finally, perhaps we should not be seduced by biventricular repair at all. Although the very long-term outcomes of the functionally univentricular circulation are clearly cause

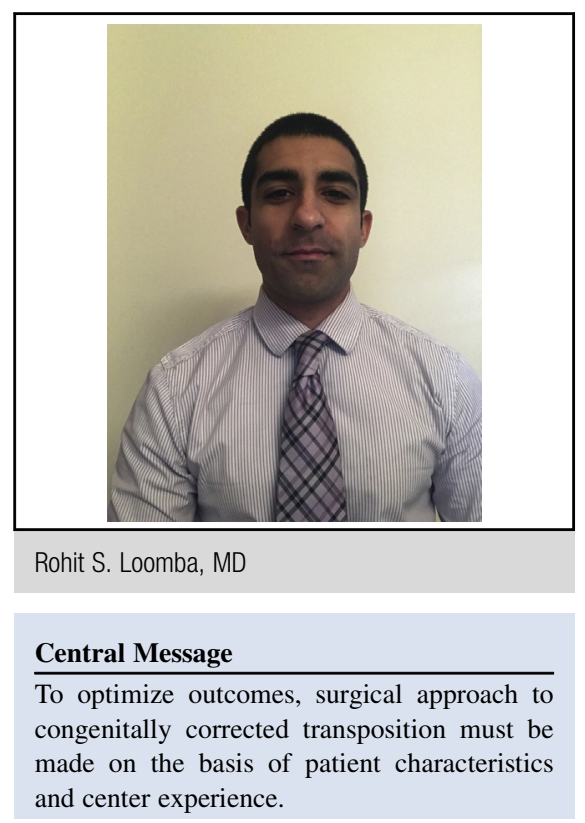

See Article page 256

for significant concern, the very low early to midterm mortality and the infrequent need for reoperation after a Fontan procedure make it worthy of consideration, particularly when the underlying anatomy makes biventricular repair unattractive (eg, straddling valves).

Consequently, as with so much of what we do, it is all about doing the right thing, for the right patient, at the right time. But that is easy to say. What is one to do with the 6-week-old patient with an intact ventricular septum, good biventricular function, and normal tricuspid valve function, for example? Some might argue that "preemptive" anatomic correction should be offered to these patients to avoid the dilemma of an "unconditioned" LV and worsening TR down the line. Maybe we should place a preemptive band to maintain conditioning of the LV and perform an anatomic repair in later infancy or early childhood? The data of Brizard and colleagues ${ }^{1}$ are quite sobering in that regard. Anatomic correction is not for the faint of heart, either literally or metaphorically. Our 6-week-old may do well without any intervention for decades, so can we yet justify the risk of a preemptive intervention?

As the experience with ccTGA increases and the body of literature grows, it has become increasingly evident 
that patient-specific characteristics and center-specific experience must guide decision making. This is a rare disease, however, and no single center will provide us with all the answers. If ever there were a condition for which we need to pool our resources as an international community to reach a better understanding of outcomes on the basis of common protocols and standardized reporting of follow-up (eg, a prescribed treatment protocol registry approach such as has proven to be successful in rare cancers), it is ccTGA.

\section{References}

1. Brizard CP, Lee A, Zannino D, Davis AM, Fricke TA, d'Udekem Y, et al Long-term results of anatomical correction for congenitally corrected transposition of the great arteries: a 19-year experience. J Thorac Cardiovasc Surg. 2017; 154:256-65.e4.

2. Adachi O, Masaki N, Kawatsu S, Yoshioka I, Masuda S, Fujiwara H, et al. Long-term results after physiologic repair for congenitally corrected transposition of the great arteries. Gen Thorac Cardiovasc Surg. 2016;64: 715-21.

3. Hraska V, Duncan BW, Mayer JE Jr, Freed M, del Nido PJ, Jonas RA. Long-term outcome of surgically treated patients with corrected transposition of the great arteries. J Thorac Cardiovasc Surg. 2005;129:182-91. 